S. W. Kim and J. B. Lee

Nagoya Math. J.

Vol. 186 (2007), 69-93

\title{
AVERAGING FORMULA FOR NIELSEN COINCIDENCE NUMBERS
}

\author{
SEUNG WON KIM AND JONG BUM LEE
}

\begin{abstract}
In this paper we study the averaging formula for Nielsen coincidence numbers of pairs of maps $(f, g): M \rightarrow N$ between closed smooth manifolds of the same dimension. Suppose that $G$ is a normal subgroup of $\Pi=\pi_{1}(M)$ with finite index and $H$ is a normal subgroup of $\Delta=\pi_{1}(N)$ with finite index such that $f_{*}(G) \subset H$ and $g_{*}(G) \subset H$. Then we investigate the conditions for which the following averaging formula holds

$$
N(f, g)=\frac{1}{[\Pi: G]} \sum_{\bar{\alpha} \in \Delta / H} N(\bar{\alpha} \bar{f}, \bar{g}),
$$

where $(\bar{f}, \bar{g}): M_{G} \rightarrow N_{H}$ is any pair of fixed liftings of $(f, g)$. We prove that the averaging formula holds when $M$ and $N$ are orientable infra-nilmanifolds of the same dimension, and when $M=N$ is a non-orientable infra-nilmanifold with holonomy group $\mathbb{Z}_{2}$ and $(f, g)$ admits a pair of liftings $(\bar{f}, \bar{g}): \bar{M} \rightarrow \bar{M}$ on the nil-covering $\bar{M}$ of $M$.
\end{abstract}

\section{$\S 1$. Introduction}

Let $f, g: X \rightarrow Y$ be a pair of maps between closed manifolds $X, Y$ with the same dimension $n$. Then we define

$$
\operatorname{Coin}(f, g)=\{x \in X \mid f(x)=g(x)\}
$$

the coincidence set of $f$ and $g$. The coincidence theory with $f, g$ is a natural extension of the fixed point theory with a self map $f: X \rightarrow X$.

If $X$ and $Y$ are orientable, then there are two well-known invariants which are the Lefschetz coincidence number $L(f, g)$ and the Nielsen coincidence number $N(f, g)$. The Lefschetz coincidence number $L(f, g)$ is an integer and $L(f, g) \neq 0$ implies the existence of a coincidence for any maps $f^{\prime}, g^{\prime}$ which are homotopic to $f, g$ respectively. The Nielsen coincidence

Received April 14, 2005.

2000 Mathematics Subject Classification: 55M20, 57S30.

Supported in part by Korea Research Foundation Grant funded by the Korean Government(MOEHRD) (KRF-2004-015-C00050 and H00021). 
number $N(f, g)$ which was defined by Schirmer [17], is a non-negative integer with the property that any two maps $f^{\prime}, g^{\prime}$ which are homotopic to $f, g$ respectively, have at least $N(f, g)$ coincidences. She showed that if $n \geq 3$, then there are two maps $f^{\prime}, g^{\prime}$, homotopic to $f, g$ respectively, such that they have exactly $N(f, g)$ coincidences. Thus the Nielsen coincidence number is much more powerful than the Lefschetz coincidence number but to compute the Nielsen coincidence number is very hard.

If $X$ and $Y$ are non-orientable, then $L(f, g)$ and $N(f, g)$ are not defined in general. In [5], Dobreńko and Jezierski define the semi-index and the generalized Nielsen coincidence number $N(f, g)$ for maps $f, g$ between any two closed smooth manifolds of the same dimension. This generalized Nielsen coincidence number is an extension of the ordinary Nielsen coincidence number. That means that if both $X$ and $Y$ are orientable, then the generalized Nielsen coincidence number $N(f, g)$ is just the same as the ordinary Nielsen coincidence number. They also show that if $n \geq 3$, then there are two maps $f^{\prime}, g^{\prime}$, homotopic to $f, g$ respectively, such that they have exactly $N(f, g)$, the generalized Nielsen coincidence number, coincidences. In the following, every Nielsen coincidence number means generalized Nielsen coincidence number.

The purpose of this paper is to investigate the relations between the Nielsen coincidence numbers of maps $(f, g): M \rightarrow N$ and those of liftings $(\bar{f}, \bar{g}): \bar{M} \rightarrow \bar{N}$, if they exist, to finite regular coverings $\bar{M}, \bar{N}$ of $M, N$. We review the necessary background about coincidence set and coincidence classes in the next section. In Section 3, we deal with some properties about the semi-index. In Section 4, we first investigate the Nielsen coincidence numbers under finite regular covering projections. These allow us to obtain the general formula for Nielsen coincidence number on closed smooth manifolds which is described at Theorem 4.6. This theorem is an extension of [13, Theorem 3.1] (see also [14, Theorem 3.4]) which concerns with Nielsen fixed point numbers on closed manifolds and is also an extension of the McCord's result in [16] which deals with only orientable manifolds. Since the Nielsen coincidence number on nilmanifolds is well understood (see [4], [6], [7], [10] and [19]) and every infra-nilmanifold admits a finite covering by a closed nilmanifold, the averaging formula becomes a practical formula on infra-nilmanifolds. At the end of this section, we show that the Nielsen coincidence number of maps between two orientable infra-nilmanifolds of the same dimension is calculated by the averaging formula. In Section 5, we also show that the averaging formula for the Nielsen coincidence number 
is valid for self maps of a non-orientable infra-nilmanifold with holonomy group $\mathbb{Z}_{2}$. Finally, we give two examples related to our results in the last section.

\section{§2. Preliminaries}

In this section, we review the necessary background about coincidence set and coincidence classes.

Let $X$ and $Y$ be compact connected polyhedra, and $(f, g): X \rightarrow Y$ be a pair of maps. Let $\Pi$ and $\Delta$ be the groups of covering transformations on the universal coverings $\widetilde{X}$ and $\widetilde{Y}$ of $X$ and $Y$, respectively.

In what follows, we will fix a pair of liftings of $(f, g): X \rightarrow Y$ on their universal coverings and denote this by $(\tilde{f}, \tilde{g}): \widetilde{X} \rightarrow \widetilde{Y}$. For the fixed pair of liftings $(\tilde{f}, \tilde{g})$, we have homomorphisms $\varphi, \psi: \Pi \rightarrow \Delta$ defined by

$$
\tilde{f} \alpha=\varphi(\alpha) \tilde{f}, \quad \tilde{g} \alpha=\psi(\alpha) \tilde{g}, \quad \alpha \in \Pi .
$$

The homomorphisms $\varphi, \psi$ define the Reidemeister action of $\Pi$ on $\Delta$ as follows:

$$
\Pi \times \Delta \longrightarrow \Delta, \quad(\gamma, \alpha) \longmapsto \psi(\gamma) \alpha \varphi(\gamma)^{-1} .
$$

Denote the set of Reidemeister classes of $\Delta$ determined by $\varphi, \psi$ by $\mathcal{R}[\varphi, \psi]$. Let $p: \widetilde{X} \rightarrow X$ be the universal covering projection. By definition, a coincidence class $\mathbb{S}$ in the coincidence set $\operatorname{Coin}(f, g)$ is a subset of Coin $(f, g)$ of the form $p(\operatorname{Coin}(\alpha \tilde{f}, \tilde{g}))$ for some $\alpha \in \Pi$. Then it is well known that the coincidence set Coin $(f, g)$ splits into a disjoint union of coincidence classes. That is,

$$
\operatorname{Coin}(f, g)=\coprod_{[\alpha] \in \mathcal{R}[\varphi, \psi]} p(\operatorname{Coin}(\alpha \tilde{f}, \tilde{g}))
$$

Let $G$ and $H$ be normal subgroups of $\Pi$ and $\Delta$ with finite index such that $\varphi(G) \subset H$ and $\psi(G) \subset H$. Denote $X_{G}=G \backslash \widetilde{X}$ and $Y_{H}=H \backslash \widetilde{Y}$. Then $X_{G}, Y_{H}$ are regular coverings of $X, Y$ such that

$$
p: \widetilde{X} \stackrel{p^{\prime}}{\longrightarrow} X_{G} \stackrel{\bar{p}}{\longrightarrow} X, \quad q: \widetilde{Y} \stackrel{q^{\prime}}{\longrightarrow} Y_{H} \stackrel{\bar{q}}{\longrightarrow} Y
$$

are covering projections. Since $\varphi(G) \subset H$ and $\psi(G) \subset H,(\tilde{f}, \tilde{g})$ induces 
the pair of liftings $(\bar{f}, \bar{g}): X_{G} \rightarrow Y_{H}$ of $(f, g)$ so that the following diagram

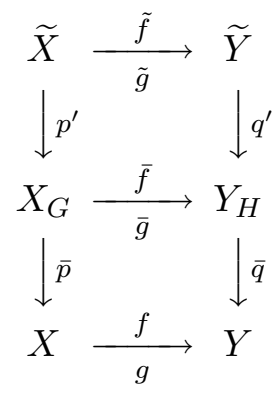

commutes.

For the pair of liftings $(\bar{f}, \bar{g})$, we have homomorphisms

$$
\begin{gathered}
\bar{\varphi}, \bar{\psi}: \Pi / G \rightarrow \Delta / H \quad \text { defined by } \bar{f} \bar{\alpha}=\bar{\varphi}(\bar{\alpha}) \bar{f}, \bar{g} \bar{\alpha}=\bar{\psi}(\bar{\alpha}) \bar{g}, \bar{\alpha} \in \Pi / G \\
\varphi^{\prime}, \psi^{\prime}: G \rightarrow H \quad \text { defined by } \tilde{f} \gamma=\varphi^{\prime}(\gamma) \tilde{f}, \tilde{g} \gamma=\psi^{\prime}(\gamma) \tilde{g}, \gamma \in G
\end{gathered}
$$

so that the following diagram commutes:

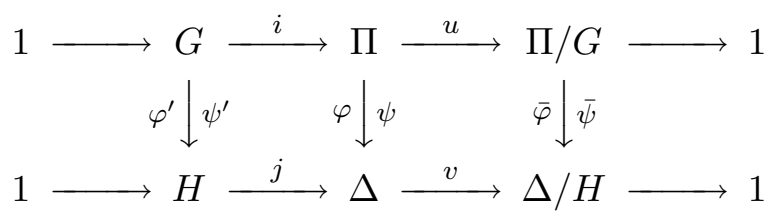

Similar to the homomorphisms $\varphi, \psi$, the homomorphisms $\varphi^{\prime}, \psi^{\prime}: G \rightarrow H$ and $\bar{\varphi}, \bar{\psi}: \Pi / G \rightarrow \Delta / H$ define the Reidemeister actions of $G$ on $H$ and $\Pi / G$ on $\Delta / H$, respectively. Denote the sets of Reidemeister classes of $H$, $\Delta / H$ determined by $\varphi^{\prime}, \psi^{\prime}, \bar{\varphi}, \bar{\psi}$ by $\mathcal{R}\left[\varphi^{\prime}, \psi^{\prime}\right], \mathcal{R}[\bar{\varphi}, \bar{\psi}]$, respectively. Then we have a short sequence of sets

$$
\mathcal{R}\left[\varphi^{\prime}, \psi^{\prime}\right] \stackrel{\hat{j}}{\longrightarrow} \mathcal{R}[\varphi, \psi] \stackrel{\hat{v}}{\longrightarrow} \mathcal{R}[\bar{\varphi}, \bar{\psi}] \longrightarrow 1
$$

where $\hat{v}$ is surjective and $\hat{v}^{-1}([\overline{1}])=\operatorname{im}(\hat{j})$ where $\overline{1}$ is the identity element of $\Delta / H$. For each $\bar{\alpha} \in \Delta / H$ and $\alpha \in v^{-1}(\bar{\alpha}), \alpha \tilde{f}$ is a lifting of $\bar{\alpha} \bar{f}$ and $f$, and $\bar{\alpha} \bar{f}$ is a lifting of $f$. They induce homomorphisms $\tau_{\alpha} \varphi, \tau_{\bar{\alpha}} \bar{\varphi}$ and $\tau_{\alpha} \varphi^{\prime}$, where $\tau_{\alpha}$ denotes conjugation by $\alpha$. In the following, for any group homomorphisms $\eta, \theta: A \rightarrow B$,

$$
\operatorname{coin}(\eta, \theta)=\{a \in A \mid \eta(a)=\theta(a)\}
$$


Then the following diagram

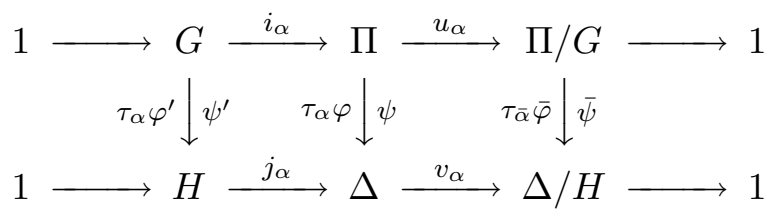

commutes, and the following sequence of groups

$$
1 \longrightarrow \operatorname{coin}\left(\tau_{\alpha} \varphi^{\prime}, \psi^{\prime}\right) \stackrel{i_{\alpha}}{\longrightarrow} \operatorname{coin}\left(\tau_{\alpha} \varphi, \psi\right) \stackrel{u_{\alpha}}{\longrightarrow} \operatorname{coin}\left(\tau_{\bar{\alpha}} \bar{\varphi}, \bar{\psi}\right)
$$

is exact, and the following sequence of sets

$$
\mathcal{R}\left[\tau_{\alpha} \varphi^{\prime}, \psi^{\prime}\right] \stackrel{\hat{j}_{\alpha}}{\longrightarrow} \mathcal{R}\left[\tau_{\alpha} \varphi, \psi\right] \stackrel{\hat{v}_{\alpha}}{\longrightarrow} \mathcal{R}\left[\tau_{\bar{\alpha}} \bar{\varphi}, \bar{\psi}\right] \longrightarrow 1
$$

satisfies that $\hat{v}_{\alpha}$ is surjective and $\hat{v}_{\alpha}^{-1}([\overline{1}])=\operatorname{im}\left(\hat{j}_{\alpha}\right)$.

If $\alpha^{\prime}=\psi(\sigma) \alpha \varphi(\sigma)^{-1}$, then $\tau_{\sigma}: \operatorname{coin}\left(\tau_{\alpha} \varphi, \psi\right) \rightarrow \operatorname{coin}\left(\tau_{\alpha^{\prime}} \varphi, \psi\right)$ is an isomorphism. In particular, if $\alpha \sim \alpha^{\prime}$, then $\left|\operatorname{coin}\left(\tau_{\alpha} \varphi, \psi\right)\right|=\left|\operatorname{coin}\left(\tau_{\alpha^{\prime}} \varphi, \psi\right)\right|$.

The following lemma is a straightforward extension of [13, Lemma 2.1].

LEMma 2.1. For each $\gamma \in H$ and $\alpha \in \Delta$, we have

(1) $\left|\hat{v}^{-1}([\bar{\alpha}])\right|=\left|\hat{v}_{\alpha}^{-1}([\overline{1}])\right|$,

(2) $|\mathcal{R}[\varphi, \psi]|=\sum_{[\bar{\alpha}] \in \mathcal{R}[\bar{\varphi}, \bar{\psi}]}\left|\hat{v}^{-1}([\bar{\alpha}])\right|=\sum_{[\bar{\alpha}] \in \mathcal{R}[\bar{\varphi}, \bar{\psi}]}\left|\hat{v}_{\alpha}^{-1}([\overline{1}])\right|$

$$
=\sum_{[\bar{\alpha}] \in \mathcal{R}[\bar{\varphi}, \bar{\psi}]}\left|\operatorname{im}\left(\hat{j}_{\alpha}\right)\right|,
$$

(3) $\left|\mathcal{R}\left[\tau_{\alpha} \varphi^{\prime}, \psi^{\prime}\right]\right|=\sum_{[\gamma] \in \operatorname{im}\left(\hat{j}_{\alpha}\right)}\left|\hat{j}_{\alpha}^{-1}([\gamma])\right|$,

(4) $\left|\hat{j}_{\alpha}^{-1}([\gamma])\right|=\left[\operatorname{coin}\left(\tau_{\bar{\alpha}} \bar{\varphi}, \bar{\psi}\right): u_{\gamma \alpha} \operatorname{coin}\left(\tau_{\gamma \alpha} \varphi, \psi\right)\right]$,

(5) $\left|\mathcal{R}\left[\tau_{\alpha} \varphi^{\prime}, \psi^{\prime}\right]\right|=\sum_{[\gamma] \in \operatorname{im}\left(\hat{j}_{\alpha}\right)}\left[\operatorname{coin}\left(\tau_{\bar{\alpha}} \bar{\varphi}, \bar{\psi}\right): u_{\gamma \alpha} \operatorname{coin}\left(\tau_{\gamma \alpha} \varphi, \psi\right)\right]$.

The coincidence classes of $(f, g)$ are labelled by the Reidemeister classes $\mathcal{R}[\varphi, \psi]$ of $(\varphi, \psi)$. We may relabel them in terms of the Reidemeister classes $\mathcal{R}[\bar{\varphi}, \bar{\psi}]$ and $\mathcal{R}\left[\tau_{\alpha} \varphi^{\prime}, \psi^{\prime}\right]$. This relabelling is useful in comparing the Nielsen number $N(f, g)$ of $(f, g)$ with the Nielsen numbers $N(\bar{\alpha} \bar{f}, \bar{g})$ of $(\bar{\alpha} \bar{f}, \bar{g})$.

Lemma 2.2. (Decomposition of the Coincidence Set) Let $f, g: X \rightarrow$ $Y$ be maps between compact connected spaces. Then

(1) $\bar{p}(\operatorname{Coin}(\bar{\alpha} \bar{f}, \bar{g}))=\coprod_{[\gamma] \in \operatorname{im}\left(\hat{j}_{\alpha}\right)} p(\operatorname{Coin}(\gamma \alpha \tilde{f}, \tilde{g}))$, 
(2) $\operatorname{Coin}(f, g)=\coprod_{[\bar{\alpha}] \in \mathcal{R}[\bar{\varphi}, \bar{\psi}]} \coprod_{[\gamma] \in \operatorname{im}\left(\hat{j}_{\alpha}\right)} p(\operatorname{Coin}(\gamma \alpha \tilde{f}, \tilde{g}))$.

Actually these results make sense for any spaces $X, Y$ with universal covering spaces.

Proof. Just like as the universal covering case, we can decompose the coincidence set Coin $(f, g)$ for the regular covering,

$$
\operatorname{Coin}(f, g)=\coprod_{[\bar{\alpha}] \in \mathcal{R}[\bar{\varphi}, \bar{\psi}]} \bar{p}(\operatorname{Coin}(\bar{\alpha} \bar{f}, \bar{g})) .
$$

For $\alpha \in \Delta$, let $\bar{\alpha}=v(\alpha) \in \Delta / H$. Also we have

$$
\operatorname{Coin}(\bar{\alpha} \bar{f}, \bar{g})=\coprod_{[\gamma] \in \mathcal{R}\left[\tau_{\alpha} \varphi^{\prime}, \psi^{\prime}\right]} p^{\prime}(\operatorname{Coin}(\gamma \alpha \tilde{f}, \tilde{g}))
$$

Thus

$$
\begin{aligned}
\bar{p}(\operatorname{Coin}(\bar{\alpha} \bar{f}, \bar{g})) & =\bigcup_{[\gamma] \in \mathcal{R}\left[\tau_{\alpha} \varphi^{\prime}, \psi^{\prime}\right]} p(\operatorname{Coin}(\gamma \alpha \tilde{f}, \tilde{g})), \\
\operatorname{Coin}(f, g) & =\coprod_{[\bar{\alpha}] \in \mathcal{R}[\bar{\varphi}, \bar{\psi}]} \bar{p}(\operatorname{Coin}(\bar{\alpha} \bar{f}, \bar{g})) \\
& =\coprod_{[\bar{\alpha}] \in \mathcal{R}[\bar{\varphi}, \bar{\psi}]} \bigcup_{[\gamma] \in \mathcal{R}\left[\tau_{\alpha} \varphi^{\prime}, \psi^{\prime}\right]} p(\operatorname{Coin}(\gamma \alpha \tilde{f}, \tilde{g})) \\
& =\text { a union of fixed point classes of } f .
\end{aligned}
$$

By Lemma 2.1, the number of different coincidence classes $p(\operatorname{Coin}(\gamma \alpha \tilde{f}$, $\tilde{g}))$ involved in $\bar{p}(\operatorname{Coin}(\bar{\alpha} \bar{f}, \bar{g}))$ is $\left|\operatorname{im}\left(\hat{j}_{\alpha}\right)\right|$. Hence

$$
\begin{aligned}
\bar{p}(\operatorname{Coin}(\bar{\alpha} \bar{f}, \bar{g})) & =\coprod_{[\gamma] \in \operatorname{im}\left(\hat{j}_{\alpha}\right)} p(\operatorname{Coin}(\gamma \alpha \tilde{f}, \tilde{g})) \\
\operatorname{Coin}(f, g) & =\coprod_{[\bar{\alpha}] \in \mathcal{R}[\bar{\varphi}, \bar{\psi}]} \coprod_{[\gamma] \in \operatorname{im}\left(\hat{j}_{\alpha}\right)} p(\operatorname{Coin}(\gamma \alpha \tilde{f}, \tilde{g}))
\end{aligned}
$$

This proves the result.

\section{$\S 3 . \quad$ Semi-index}

We first recall the definition of the semi-index [5]. Let $V_{1}, V_{2}$ be finitedimensional real vector spaces, and let $\alpha, \beta$ be the orientations determined 
by the ordered bases $\left\{v_{1}, \ldots, v_{k}\right\}$ and $\left\{v_{k+1}, \ldots, v_{k+\ell}\right\}$ of $V_{1}$ and $V_{2}$, respectively. Then $\alpha \wedge \beta$ denotes the orientation of $V_{1} \oplus V_{2}$ determined by the ordered basis $\left\{v_{1}, \ldots, v_{k}, v_{k+1}, \ldots, v_{k+\ell}\right\}$.

The pair of maps $(f, g): M \rightarrow N$ between smooth manifolds is called transversal if both $f$ and $g$ are smooth maps and, for any $x \in \operatorname{Coin}(f, g)$, the difference of the induced tangent maps

$$
T_{x} f-T_{x} g: T_{x} M \longrightarrow T_{f(x)} N
$$

is an epimorphism.

It is well-known (cf. [9, Lemma 5.1.5]) that any map on smooth manifolds can be approximated by smooth maps homotopic to it. Moreover, the following is an easy consequence of "Transversality Homotopy Theorem" $([8])$ :

LEMma 3.1. Any pair of smooth maps $f, g: M \rightarrow N$ is homotopic to a transversal pair.

LEMma 3.2. Two smooth maps $f, g: M \rightarrow N$ are transversal if and only if the graphs $G(f)$ and $G(g)$ of $f$ and $g$ are transversal as the submanifolds of $M \times N$, that is, for any $x \in M$ with $f(x)=y=g(x)$, the "transversality assumption" holds:

$$
T_{(x, y)} G(f)+T_{(x, y)} G(g)=T_{(x, y)} M \times N .
$$

Proof. Since the tangent space to $G(f)$ at the point $(x, f(x))$ is the graph of $T_{x} f: T_{x} M \rightarrow T_{f(x)} N$, it is sufficient to show the following: For a pair of linear maps $\phi, \psi: V \rightarrow W, \phi-\psi: V \rightarrow W$ is an epimorphism if and only if $G(\phi)+G(\psi)=V \oplus W$.

Suppose $G(\phi)+G(\psi)=V \oplus W$. Then for any $w \in W$, there are $v_{1}, v_{2} \in V$ such that

$$
(0, w)=\left(v_{1}, \phi\left(v_{1}\right)\right)+\left(v_{2}, \psi\left(v_{2}\right)\right)
$$

Thus $v_{2}=-v_{1}$ and $w=\phi\left(v_{1}\right)-\psi\left(v_{1}\right)$. Conversely suppose $\phi-\psi: V \rightarrow W$ is an epimorphism. For any $(v, w) \in V \oplus W$, since $\phi-\psi$ is an epimorphism, there is $v_{1} \in V$ such that $w-\psi\left(v_{1}\right)=\phi\left(v_{1}\right)-\psi\left(v_{1}\right)$. This show that $(v, w)=\left(v_{1}, \phi\left(v_{1}\right)\right)+\left(v-v_{1}, \psi\left(v-v_{1}\right)\right) \in V \oplus W$. 
Remark 3.3. For $f, g: M \rightarrow N$, let $F, G: M \rightarrow M \times N$ be given by

$$
F(x)=(x, f(x)), \quad G(x)=(x, g(x))
$$

and let $h: M \rightarrow N \times N$ be given by $h(x)=(f(x), g(x))$. Then it is easy to see that $f$ is transversal to $g$ if and only if $F$ is transversal to $G$, equivalently $h$ is transversal to the inclusion $\Delta_{N} \rightarrow N \times N$ in the sense of [3, Definition II.15.1].

If two smooth maps $f, g: M \rightarrow N$ are transversal, then by [8, Theorem 1.5], $G(f) \cap G(g)$ is a submanifold of $M \times N$. Since Coin $(f, g)$ is diffeomorphic to $G(f) \cap G(g)$, Coin $(f, g)$ is a submanifold of $M$. Moreover, the dimension of $\operatorname{Coin}(f, g)$ equals $\operatorname{dim} M-\operatorname{dim} N$. For,

$$
\begin{aligned}
\operatorname{dim} \operatorname{Coin}(f, g) & =\operatorname{dim} G(f) \cap G(g) \\
& =\operatorname{dim} T_{(x, y)}(G(f) \cap G(g)) \\
& =\operatorname{dim} T_{(x, y)} G(f) \cap T_{(x, y)} G(g) \\
& =\operatorname{dim} G\left(T_{x} f\right) \cap G\left(T_{x} g\right) \\
& =\operatorname{dim} \operatorname{Coin}\left(T_{x} f, T_{x} g\right) \\
& =\operatorname{dim} \operatorname{ker}\left(T_{x} f-T_{x} g\right) \\
& =\operatorname{dim} M-\operatorname{dim} N,
\end{aligned}
$$

where $x$ is any element of $M$ with $f(x)=y=g(x)$.

In what follows, we assume that $M, N$ are closed smooth connected manifolds of the same dimension, and $(f, g): M \rightarrow N$ is a transversal pair. Then Coin $(f, g)$ is a finite set, and for any $x \in \operatorname{Coin}(f, g)$ with $f(x)=y=$ $g(x)$

$$
T_{(x, y)} G(f) \oplus T_{(x, y)} G(g)=T_{x} M \oplus T_{y} N .
$$

We fix local orientations, $\alpha_{x}$ and $\beta_{x}$, of the graphs $G(f)$ and $G(g)$, respectively, at the point $(x, y)$. Then $\gamma_{x}=\alpha_{x} \wedge \beta_{x}$ is a local orientation of $M \times N$ at this point.

Definition 3.4. We say that $x, x^{\prime} \in \operatorname{Coin}(f, g)$ reduce one another if there exists a path $\omega$ in $M$ from $x$ to $x^{\prime}$ such that

(1) $f \omega \simeq g \omega$ (relative to endpoints), and thus $x$ and $x^{\prime}$ belong to the same coincidence class, 
(2) if $\alpha_{x^{\prime}}$ and $\beta_{x^{\prime}}$ denote the translations of $\alpha_{x}$ and $\beta_{x}$ in $G(f)$ and $G(g)$ along the paths $(\omega, f \omega)$ and $(\omega, g \omega)$, respectively, and if $\gamma_{x^{\prime}}$ denotes the translation of $\gamma_{x}$ in $M \times N$ along the path $(\omega, f \omega)$, then

$$
\alpha_{x^{\prime}} \wedge \beta_{x^{\prime}}=-\gamma_{x^{\prime}} .
$$

In this case, we say that the path $\omega$ reverses orientation in graphs.

Let $A \subset \operatorname{Coin}(f, g)$ be any subset. It may be represented as

$$
A=\left\{a_{1}, b_{1}, \ldots, a_{k}, b_{k} ; c_{1}, \ldots, c_{s}\right\}
$$

where $a_{i}, b_{i}$ reduce one another and $c_{i}, c_{j}$ do not reduce one another for any $i \neq j$. We call the elements $\left\{c_{1}, \ldots, c_{s}\right\}$ free in this representation of $A \subset \operatorname{Coin}(f, g)$. The number of free elements does not depend on the representation of $A$ ([5, Lemma 1.3]).

Lemma 3.5. ([5, Lemma 2.1]) Let $\bar{x}, \bar{y} \in p^{\prime}\left(\operatorname{Coin}\left(\gamma^{\prime} \alpha \tilde{f}, \tilde{g}\right)\right)$ and let $\bar{\omega}$ be a path from $\bar{x}$ to $\bar{y}$ such that $\bar{f} \bar{\omega} \simeq \bar{g} \bar{\omega}$. Then for $x=\bar{p} \bar{x}, y=\bar{p} \bar{y}, \omega=\bar{p} \bar{\omega}$, we have $x, y \in p(\operatorname{Coin}(\gamma \alpha \tilde{f}, \tilde{g}))$ and $\omega$ is a path from $x$ to $y$ satisfying $f \omega \simeq g \omega$. Under these assumptions $\bar{\omega}$ reverses orientations in graphs if and only if so does $\omega$.

Lemma 3.6. ([5, Corollary 2.2]) If the points $x, y \in p(\operatorname{Coin}(\gamma \alpha \tilde{f}, \tilde{g}))$ reduce one another, then $\bar{p}^{-1}(\{x, y\}) \cap p^{\prime}\left(\operatorname{Coin}\left(\gamma^{\prime} \alpha \tilde{f}, \tilde{g}\right)\right)$ splits into pairs of points reducing one another.

Definition 3.7. A coincidence point $x \in \operatorname{Coin}(f, g)$ is called selfreducing if it reduces itself. A coincidence class is called defective if it contains a self-reducing point.

Lemma 3.8. ([11, Lemma 2.2]) For $x \in p(\operatorname{Coin}(\alpha \tilde{f}, \tilde{g})), x$ is self-reducing if and only if

$$
\operatorname{coin}\left(\tau_{\alpha} \varphi, \psi\right) \cap \Pi^{+} \neq \operatorname{coin}\left(\tau_{\alpha} \varphi, \psi\right) \cap\left(\tau_{\alpha} \varphi\right)^{-1}\left(\Delta^{+}\right),
$$

where $\Pi^{+}$and $\Delta^{+}$are the subgroups of $\Pi$ and $\Delta$, respectively, which consist of all orientation preserving elements. In particular, a defective coincidence class consists of self-reducing points. 
Lemma 3.9. Suppose that $x \in p(\operatorname{Coin}(\alpha \tilde{f}, \tilde{g}))$ and $\bar{x} \in p^{\prime}\left(\operatorname{Coin}\left(\alpha^{\prime} \tilde{f}, \tilde{g}\right)\right)$ where $\bar{p}(\bar{x})=x$ and $\alpha^{\prime} \in[\alpha] \in \mathcal{R}[\varphi, \psi]$. Then the following are equivalent:

(1) There is $\gamma \in \operatorname{coin}\left(\tau_{\alpha} \varphi, \psi\right)$ such that $\bar{x}$ and $\bar{\gamma} \bar{x}$ reduce one another.

(2) There is $\gamma \in \operatorname{coin}\left(\tau_{\alpha} \varphi, \psi\right)$ such that $\operatorname{sgn}\left(\tau_{\bar{\alpha}} \bar{\varphi}(\bar{\gamma})\right) \cdot \operatorname{sgn}(\bar{\gamma})=-1$.

(3) $u_{\alpha} \operatorname{coin}\left(\tau_{\alpha} \varphi, \psi\right) \cap \Pi^{+} G / G \neq u_{\alpha} \operatorname{coin}\left(\tau_{\alpha} \varphi, \psi\right) \cap\left(\tau_{\bar{\alpha}} \bar{\varphi}\right)^{-1}\left(\Delta^{+} H / H\right)$.

In this case, $x$ is self-reducing.

Proof. Suppose that $\bar{x}$ and $\bar{\gamma} \bar{x}$ reduce one another. Then there exists a path $\bar{\omega}$ from $\bar{x}$ to $\bar{\gamma} \bar{x}$ so that $\bar{\omega}$ reverses orientation in graphs and $\bar{\alpha}^{\prime} \bar{f} \bar{\omega} \simeq \bar{h} \bar{\omega}$. Since $\bar{\omega}$ is a path from $\bar{x}$ to $\bar{\gamma} \bar{x}$, the loop $p \bar{\omega}$ at $x$ represents an element $\gamma \in \Pi$ so that it projects to $\bar{\gamma} \in \Pi / G$. Let $\tilde{\omega}$ be the lift of $\bar{\omega}$ starting at $\tilde{x}$. Then $\tilde{\omega}(1)=\gamma \tilde{x}$ and $p^{\prime}(\gamma \tilde{x})=\bar{x}$. Since $\bar{\alpha}^{\prime} \bar{f} \bar{\omega} \simeq \bar{h} \bar{\omega}, \alpha^{\prime} \tilde{f} \tilde{\omega} \simeq \tilde{h} \tilde{\omega}$ and hence $\gamma \tilde{x} \in$ $\operatorname{Coin}\left(\alpha^{\prime} \tilde{f}, \tilde{h}\right)$. This implies that $\gamma \in \operatorname{coin}\left(\tau_{\alpha^{\prime}} \varphi, \psi\right)$ and $\bar{\alpha}^{\prime} \bar{f} \bar{\omega}(1)=\bar{\alpha}^{\prime} \bar{f}(\bar{\gamma} \bar{x})=$ $\tau_{\bar{\alpha}^{\prime}} \bar{\varphi}(\bar{\gamma}) \cdot \bar{\alpha}^{\prime} \bar{f}(\bar{x})=\tau_{\bar{\alpha}^{\prime}} \bar{\varphi}(\bar{\gamma}) \cdot \bar{\alpha}^{\prime} \bar{f} \bar{\omega}(0)$. Thus the path $\bar{\alpha}^{\prime} \bar{f} \bar{\omega}$ represents the element $\tau_{\bar{\alpha}^{\prime}} \bar{\varphi}(\bar{\gamma}) \in \Delta / H$. Since $\bar{\omega}$ is graph-orientation-reversing, it follows that $\operatorname{sgn}\left(\tau_{\bar{\alpha}^{\prime}} \bar{\varphi}(\bar{\gamma})\right) \cdot \operatorname{sgn}(\bar{\gamma})=-1$. Recalling that $\alpha^{\prime} \in[\alpha] \in \mathcal{R}[\varphi, \psi]$, we see that $\operatorname{sgn}\left(\tau_{\bar{\alpha}^{\prime}} \bar{\varphi}(\bar{\gamma})\right)=\operatorname{sgn}\left(\tau_{\bar{\alpha}} \bar{\varphi}(\bar{\gamma})\right)$. Therefore, $\operatorname{sgn}\left(\tau_{\bar{\alpha}} \bar{\varphi}(\bar{\gamma})\right) \cdot \operatorname{sgn}(\bar{\gamma})=-1$. This is equivalent to that either $\tau_{\bar{\alpha}} \bar{\varphi}(\bar{\gamma}) \notin \Delta^{+} H / H$ or $\bar{\gamma} \notin \Pi^{+} G / G$.

For the converse, we take a path from $\bar{x}$ to $\bar{\gamma} \bar{x}$ which represents the element $\bar{\gamma}$. Since $\operatorname{sgn}\left(\tau_{\bar{\alpha}^{\prime}} \bar{\varphi}(\bar{\gamma})\right) \cdot \operatorname{sgn}(\bar{\gamma})=-1$, it follows that the path $\bar{\omega}$ is graph-orientation-reversing.

In the course of the proof, we have $\gamma \in \operatorname{coin}\left(\tau_{\alpha^{\prime}} \varphi, \psi\right)$ which projects to $\bar{\gamma}$. Since $\operatorname{sgn}\left(\tau_{\bar{\alpha}^{\prime}} \bar{\varphi}(\bar{\gamma})\right) \cdot \operatorname{sgn}(\bar{\gamma})=-1$, it follows that

$$
\operatorname{coin}\left(\tau_{\alpha^{\prime}} \varphi, \psi\right) \cap \Pi^{+} \neq \operatorname{coin}\left(\tau_{\alpha^{\prime}} \varphi, \psi\right) \cap\left(\tau_{\alpha^{\prime}} \varphi\right)^{-1}\left(\Delta^{+}\right) .
$$

Recalling $\alpha^{\prime} \in[\alpha]$, we see that the subgroups $\operatorname{coin}\left(\tau_{\alpha} \varphi, \psi\right)$ and $\operatorname{coin}\left(\tau_{\alpha^{\prime}} \varphi, \psi\right)$ are conjugate. Furthermore, since $\Delta^{+}$is a normal subgroup of $\Delta$, it follows that $\left(\tau_{\alpha} \varphi\right)^{-1}\left(\Delta^{+}\right)=\left(\tau_{\alpha^{\prime}} \varphi\right)^{-1}\left(\Delta^{+}\right)$. Therefore,

$$
\operatorname{coin}\left(\tau_{\alpha} \varphi, \psi\right) \cap \Pi^{+} \neq \operatorname{coin}\left(\tau_{\alpha} \varphi, \psi\right) \cap\left(\tau_{\alpha} \varphi\right)^{-1}\left(\Delta^{+}\right) .
$$

Now Lemma 3.8 implies that $x$ is self-reducing.

Definition 3.10. The semi-index of $A \subset \operatorname{Coin}(f, g)$ is the number of free elements in any representation of $A$ and is denoted by $\mid$ ind $\mid(f, g ; A)$.

Note that the definition of semi-index for a pair of transversal maps is extended over arbitrary pair of maps ([5, Lemma 1.4]). The semi-index has the following properties: 
(i) Existence of coincidences. If $\mid$ ind $\mid(f, g ; \mathbb{S}) \neq 0$, then $\operatorname{Coin}(f, g) \neq \varnothing$ where $\mathbb{S}$ is a coincidence class in Coin $(f, g)$.

(ii) Homotopy invariance. If $\left(f_{0}, g_{0}\right) \stackrel{F, G}{\simeq}\left(f_{1}, g_{1}\right): M \rightarrow N$, then

$$
|\operatorname{ind}|\left(f_{0}, g_{0} ; \mathbb{S}_{0}\right)=|\operatorname{ind}|\left(f_{1}, g_{1} ; \mathbb{S}_{1}\right)
$$

where a coincidence class $\mathbb{S}_{0}$ corresponds to the coincidence class $\mathbb{S}_{1}$ along the given homotopy.

(iii) Additivity. For any coincidence classes $\mathbb{S}_{i}$ in $\operatorname{Coin}(f, g)$,

$$
\mid \text { ind }\left|\left(f, g ; \bigcup \mathbb{S}_{i}\right)=\sum_{i}\right| \operatorname{ind} \mid\left(f, g ; \mathbb{S}_{i}\right)
$$

Definition 3.11. Let $(f, g): M \rightarrow N$ be maps. A coincidence class $\mathbb{S} \subset \operatorname{Coin}(f, g)$ is called essential if $|\operatorname{ind}|(f, g ; \mathbb{S}) \neq 0$. Otherwise, it is called inessential. We define the Nielsen coincidence number of $(f, g)$ as the number of its essential classes, and we denote it by $N(f, g)$.

It is known from [5, Lemma 1.6] that for any pair of maps $(f, g)$ : $M \rightarrow N$ between orientable manifolds of the same dimension and for any coincidence class $\mathbb{S} \subset \operatorname{Coin}(f, g)$,

$$
|\operatorname{ind}|(f, g ; \mathbb{S})=|\operatorname{ind}(f, g ; \mathbb{S})|
$$

where the right side denotes the absolute value of the ordinary coincidence index. Therefore the above definitions relative to semi-index generalize the ordinary definitions relative to classical index. In particular, the Nielsen coincidence numbers for orientable manifolds are the same as the ordinary Nielsen coincidence numbers where the ordinary Nielsen coincidence number is the number of coincidence classes which have non-zero ordinary coincidence indices.

\section{§4. Nielsen coincidence numbers for covering spaces}

Let $M$ and $N$ be closed smooth connected manifolds of the same dimension, and $(f, g): M \rightarrow N$ a pair of transversal maps. Let $\Pi$ and $\Delta$ be the groups of covering transformations on the universal coverings $\widetilde{M}$ and $\widetilde{N}$ of $M$ and $N$, respectively. Let $(\tilde{f}, \tilde{g}): \widetilde{M} \rightarrow \widetilde{N}$ be a fixed pair of liftings of $(f, g)$. We denote the homomorphisms induced by $(\tilde{f}, \tilde{g})$ by

$$
\varphi, \psi: \Pi \longrightarrow \Delta \text {. }
$$


Suppose $G$ and $H$ are normal subgroups of $\Pi$ and $\Delta$ with finite index such that $\varphi(G) \subset H$ and $\psi(G) \subset H$. Then the fixed $(\tilde{f}, \tilde{g})$ of $(f, g)$ induces the pair of liftings $(\bar{f}, \bar{g}): M_{G} \rightarrow N_{H}$ of $(f, g)$ so that the following diagram

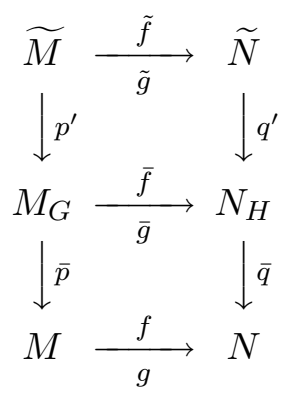

commutes.

Now we compare the Nielsen coincidence number $N(f, g)$ of $(f, g)$ with the Nielsen coincidence numbers $N(\bar{\alpha} \bar{f}, \bar{g})$ of the pair of liftings $(\bar{\alpha} \bar{f}, \bar{g})$, $(\bar{\alpha} \in \Delta / H)$, of $(f, g)$. First, we consider the relation of coincidence class of $(f, g)$ and coincidence class of $(\bar{\alpha} \bar{f}, \bar{g})$.

LEMMA 4.1.

$$
\bar{p}^{-1} p(\operatorname{Coin}(\gamma \alpha \tilde{f}, \tilde{g}))=\bigcup_{\gamma^{\prime} \in[\gamma]} p^{\prime}\left(\operatorname{Coin}\left(\gamma^{\prime} \alpha \tilde{f}, \tilde{g}\right)\right)
$$

where $[\gamma] \in \mathcal{R}\left[\tau_{\alpha} \varphi, \psi\right]$.

Proof. Noting that

$$
\begin{aligned}
\bar{p} p^{\prime}\left(\operatorname{Coin}\left(\psi(\sigma) \gamma \alpha \varphi(\sigma)^{-1} \alpha^{-1} \cdot \alpha \tilde{f}, \tilde{g}\right)\right) & =p\left(\operatorname{Coin}\left(\psi(\sigma) \cdot \gamma \alpha \cdot \varphi(\sigma)^{-1} \tilde{f}, \tilde{g}\right)\right) \\
& =p(\operatorname{Coin}(\gamma \alpha \tilde{f}, \tilde{g})),
\end{aligned}
$$

we have $\bar{p}^{-1} p(\operatorname{Coin}(\gamma \alpha \tilde{f}, \tilde{g})) \supseteq \bigcup_{\gamma^{\prime} \in[\gamma]} p^{\prime}\left(\operatorname{Coin}\left(\gamma^{\prime} \alpha \tilde{f}, \tilde{g}\right)\right)$.

Let $\bar{x} \in \bar{p}^{-1} p(\operatorname{Coin}(\gamma \alpha \tilde{f}, \tilde{g}))$. Then $\bar{p}(\bar{x})=p(\tilde{x})$ for some $\tilde{x} \in \operatorname{Coin}(\gamma \alpha \tilde{f}$, $\tilde{g})$. Then there exists $\bar{\sigma} \in \Delta / H$ such that $\bar{x}=\bar{\sigma} \cdot p^{\prime}(\tilde{x})$. Let $\sigma \in v^{-1}(\bar{\sigma})$ and $\tilde{y}=\sigma \tilde{x}$. Then $\sigma^{-1} \tilde{y}=\tilde{x} \in \operatorname{Coin}(\gamma \alpha \tilde{f}, \tilde{g})$ and $p^{\prime}(\tilde{y})=\bar{x} ; \tilde{y} \in \operatorname{Coin}(\psi(\sigma) \cdot \gamma \alpha$. $\left.\varphi(\sigma)^{-1} \tilde{f}, \tilde{g}\right)$. Thus $\bar{x} \in p^{\prime}\left(\operatorname{Coin}\left(\gamma^{\prime} \alpha \tilde{f}, \tilde{g}\right)\right)$, where $\gamma^{\prime}=\psi(\sigma) \gamma \tau_{\alpha} \varphi(\sigma)^{-1}$ and hence $\bar{p}^{-1} p(\operatorname{Coin}(\gamma \alpha \tilde{f}, \tilde{g})) \subseteq \bigcup_{\gamma^{\prime} \in[\gamma]} p^{\prime}\left(\operatorname{Coin}\left(\gamma^{\prime} \alpha \tilde{f}, \tilde{g}\right)\right)$. 
Lemma 4.2. Let $\gamma \in H$ and $\alpha \in \Delta$. Then for any $\gamma^{\prime} \in[\gamma](\in$ $\left.\mathcal{R}\left[\tau_{\alpha} \varphi, \psi\right]\right)$,

$$
\begin{aligned}
\left|p^{\prime}\left(\operatorname{Coin}\left(\gamma^{\prime} \alpha \tilde{f}, \tilde{g}\right)\right)\right| & =\left|u_{\gamma^{\prime} \alpha} \operatorname{coin}\left(\tau_{\gamma^{\prime} \alpha} \varphi, \psi\right)\right| \cdot|p(\operatorname{Coin}(\gamma \alpha \tilde{f}, \tilde{g}))| \\
& =\left|u_{\gamma \alpha} \operatorname{Coin}\left(\tau_{\gamma \alpha} \varphi, \psi\right)\right| \cdot|p(\operatorname{Coin}(\gamma \alpha \tilde{f}, \tilde{g}))| \\
& =\left|p^{\prime}(\operatorname{Coin}(\gamma \alpha \tilde{f}, \tilde{g}))\right| .
\end{aligned}
$$

In particular, the number of different coincidence classes $p^{\prime}\left(\operatorname{Coin}\left(\gamma^{\prime} \alpha \tilde{f}, \tilde{g}\right)\right)$ involved in $\bar{p}^{-1} p(\operatorname{Coin}(\gamma \alpha \tilde{f}, \tilde{g}))$ is the index $\left[\Pi / G: u_{\gamma \alpha} \operatorname{coin}\left(\tau_{\gamma \alpha} \varphi, \psi\right)\right]$.

Proof. Let $\gamma \in H$ and $\alpha \in \Delta$, and take any $[\gamma] \in \mathcal{R}\left[\tau_{\alpha} \varphi, \psi\right]$. Then $\Pi$ acts on $[\gamma]$ as follows: $\sigma \cdot \gamma=\psi(\sigma) \gamma \tau_{\alpha} \varphi(\sigma)^{-1}$. This induces the $\Pi$ action on the set of coincidence classes $\left\{p^{\prime}\left(\operatorname{Coin}\left(\gamma^{\prime} \alpha \tilde{f}, \tilde{g}\right)\right) \mid \gamma^{\prime} \in[\gamma]\right\}$ :

$$
\begin{aligned}
\left(\sigma, p^{\prime}\left(\operatorname{Coin}\left(\gamma^{\prime} \alpha \tilde{f}, \tilde{g}\right)\right)\right) \longmapsto & p^{\prime}\left(\operatorname{Coin}\left(\left(\sigma \cdot \gamma^{\prime}\right) \alpha \tilde{f}, \tilde{g}\right)\right) \\
& =p^{\prime}\left(\operatorname{Coin}\left(\left(\psi(\sigma) \gamma^{\prime} \tau_{\alpha} \varphi(\sigma)^{-1}\right) \alpha \tilde{f}, \tilde{g}\right)\right) .
\end{aligned}
$$

Let $\sigma \in G$. If $\gamma^{\prime} \in H$, then $\gamma^{\prime} \sim \psi(\sigma) \gamma^{\prime} \tau_{\alpha} \varphi(\sigma)^{-1}$. Thus $p^{\prime}\left(\operatorname{Coin}\left(\gamma^{\prime} \alpha \tilde{f}, \tilde{g}\right)\right)=$ $p^{\prime}\left(\operatorname{Coin}\left(\psi(\sigma) \gamma^{\prime} \alpha \varphi(\sigma)^{-1} \tilde{f}, \tilde{g}\right)\right)$. If $\gamma^{\prime} \notin H$, write $\gamma^{\prime}=\gamma_{0} \beta$ where $\gamma_{0} \in H$. Then

$$
\begin{aligned}
\left(\psi(\sigma) \gamma^{\prime} \tau_{\alpha} \varphi(\sigma)^{-1}\right) \alpha \tilde{f} & =\left(\psi(\sigma) \gamma_{0} \beta \alpha \varphi(\sigma)^{-1} \alpha^{-1}\right) \alpha \tilde{f} \\
& =\left(\psi(\sigma) \gamma_{0} \beta \alpha \varphi(\sigma)^{-1} \alpha^{-1} \beta^{-1}\right) \beta \alpha \tilde{f} \\
& =\left(\psi(\sigma) \gamma_{0} \tau_{\beta \alpha} \varphi(\sigma)^{-1}\right) \beta \alpha \tilde{f} \\
& \sim\left(\gamma_{0}\right) \beta \alpha \tilde{f} \\
& =\gamma^{\prime} \alpha \tilde{f} .
\end{aligned}
$$

Therefore if $\sigma \in G$, then $p^{\prime}\left(\operatorname{Coin}\left(\gamma^{\prime} \alpha \tilde{f}, \tilde{g}\right)\right)=p^{\prime}\left(\operatorname{Coin}\left(\psi(\sigma) \gamma^{\prime} \tau_{\alpha} \varphi(\sigma)^{-1} \alpha \tilde{f}, \tilde{g}\right)\right)$. This implies that we have an action:

$$
\Pi / G \times\left\{p^{\prime}\left(\operatorname{Coin}\left(\gamma^{\prime} \alpha \tilde{f}, \tilde{g}\right)\right) \mid \gamma^{\prime} \in[\gamma]\right\} \longrightarrow\left\{p^{\prime}\left(\operatorname{Coin}\left(\gamma^{\prime} \alpha \tilde{f}, \tilde{g}\right)\right) \mid \gamma^{\prime} \in[\gamma]\right\},
$$

which is transitive. The isotropy subgroup at $p^{\prime}(\operatorname{Coin}(\gamma \alpha \tilde{f}, \tilde{g}))$ is

$$
\begin{aligned}
\left\{\bar{\sigma} \in \Pi / G \mid \gamma=\psi(\sigma) \gamma \tau_{\alpha} \varphi(\sigma)^{-1}\right\} & =\left\{\bar{\sigma} \in \Pi / G \mid \sigma \in \operatorname{coin}\left(\tau_{\gamma \alpha} \varphi, \psi\right)\right\} \\
& =u_{\gamma \alpha} \operatorname{coin}\left(\tau_{\gamma \alpha} \varphi, \psi\right)
\end{aligned}
$$

Hence the number of different coincidence classes $p^{\prime}\left(\operatorname{Coin}\left(\gamma^{\prime} \alpha \tilde{f}, \tilde{g}\right)\right)$ involved in $\bar{p}^{-1} p(\operatorname{Coin}(\gamma \alpha \tilde{f}, \tilde{g}))$ is the index $\left[\Pi / G: u_{\gamma \alpha} \operatorname{coin}\left(\tau_{\gamma \alpha} \varphi, \psi\right)\right]$. 
This Lemma amounts to saying for each $x \in p(\operatorname{Coin}(\gamma \alpha \tilde{f}, \tilde{g}))$, there are $\left|u_{\gamma \alpha} \operatorname{coin}\left(\tau_{\gamma \alpha} \varphi, \psi\right)\right|$ points in each $p^{\prime}\left(\operatorname{Coin}\left(\gamma^{\prime} \alpha \tilde{f}, \tilde{g}\right)\right) \cap \bar{p}^{-1}(x)$.

Corollary 4.3.

$$
\bar{p}^{-1} p(\operatorname{Coin}(\gamma \alpha \tilde{f}, \tilde{g}))=\coprod_{\left[\Pi / G: u_{\gamma \alpha} \operatorname{coin}\left(\tau_{\gamma \alpha} \varphi, \psi\right)\right]} p^{\prime}\left(\operatorname{Coin}\left(\gamma^{\prime} \alpha \tilde{f}, \tilde{g}\right)\right) .
$$

Proof. Follows from Lemma 4.1 and Lemma 4.2.

Definition 4.4. Let $\gamma \in H, \alpha \in \Delta$ and $\gamma^{\prime} \in[\gamma] \in \mathcal{R}\left[\tau_{\alpha} \varphi, \psi\right]$. Define $\epsilon_{\gamma^{\prime} \alpha}^{\prime}$ and $\epsilon_{\gamma \alpha}$ as follows:

$\epsilon_{\gamma^{\prime} \alpha}^{\prime}= \begin{cases}1, & p^{\prime}\left(\operatorname{Coin}\left(\gamma^{\prime} \alpha \tilde{f}, \tilde{g}\right)\right) \text { is an essential coincidence class of }(\bar{\alpha} \bar{f}, \bar{g}) ; \\ 0, & p^{\prime}\left(\operatorname{Coin}\left(\gamma^{\prime} \alpha \tilde{f}, \tilde{g}\right)\right) \text { is an inessential coincidence class of }(\bar{\alpha} \bar{f}, \bar{g}),\end{cases}$

$\epsilon_{\gamma \alpha}= \begin{cases}1, & p(\operatorname{Coin}(\gamma \alpha \tilde{f}, \tilde{g})) \text { is an essential coincidence class of }(f, g) ; \\ 0, & p(\operatorname{Coin}(\gamma \alpha \tilde{f}, \tilde{g})) \text { is an inessential coincidence class of }(f, g) .\end{cases}$

Remark 4.5. Recall that the coincidence class $p(\operatorname{Coin}(\gamma \alpha \tilde{f}, \tilde{g}))$ is essential if any of the covering coincidence classes $p^{\prime}\left(\operatorname{Coin}\left(\gamma^{\prime} \alpha \tilde{f}, \tilde{g}\right)\right)$ is essential. Therefore, from Corollary 4.3, we have the following:

$$
\sum_{\left[\Pi / G: u_{\gamma \alpha} \operatorname{coin}\left(\tau_{\gamma \alpha} \varphi, \psi\right)\right]} \epsilon_{\gamma^{\prime} \alpha}^{\prime} \leq\left[\Pi / G: u_{\gamma \alpha} \operatorname{coin}\left(\tau_{\gamma \alpha} \varphi, \psi\right)\right] \cdot \epsilon_{\gamma \alpha},
$$

and if $u_{\gamma \alpha} \operatorname{coin}\left(\tau_{\gamma \alpha} \varphi, \psi\right)=\{1\}$, then, by Lemma 3.5 and Lemma 3.6, the equality occurs.

In the case that the ordinary Nielsen coincidence numbers are defined, McCord proves in [16, Corollary 5.10] that if $\operatorname{coin}\left(\tau_{\alpha} \varphi, \psi\right) \subset G$ for each $\alpha \in \Delta$ with $p(\operatorname{Coin}(\alpha \tilde{f}, \tilde{g}))$ an essential coincidence class, then

$$
N(f, g)=\frac{1}{[\Pi: G]} \sum_{\bar{\alpha} \in \Delta / H} N(\bar{\alpha} \bar{f}, \bar{g}) .
$$

When $f, g: M \rightarrow N$ are maps on the non-orientable manifolds and they admit liftings on the orientable double coverings of $M, N$, Dobreńko and Jezierski prove in [5, Theorem 2.5] that if $\operatorname{coin}\left(\tau_{\alpha} \varphi, \psi\right) \subset G$ for each $\alpha \in \Delta$ with $p(\operatorname{Coin}(\alpha \tilde{f}, \tilde{g}))$ an essential coincidence class, then

$$
N(f, g)=\frac{1}{2}(N(\bar{f}, \bar{g})+N(\bar{\alpha} \bar{f}, \bar{g})) .
$$


We may ask whether Dobreńko and Jezierski's result is true in case when the pair of maps $(f, g)$ admit liftings on some regular coverings. In Theorem 4.6 below, we prove that McCord's result and the question related to Dobreńko and Jezierski's result above are not only necessary but also sufficient.

Theorem 4.6. Let $(f, g): M \rightarrow N$ be a pair of maps between closed smooth connected manifolds of the same dimension. Suppose $G$ is a normal subgroup of $\Pi=\pi_{1}(M)$ with finite index and $H$ is a normal subgroup of $\Delta=\pi_{1}(N)$ with finite index such that $f_{*}(G) \subset H$ and $g_{*}(G) \subset H$. Then

$$
N(f, g) \geq \frac{1}{[\Pi: G]} \sum_{\bar{\alpha} \in \Delta / H} N(\bar{\alpha} \bar{f}, \bar{g}),
$$

and equality occurs if and only if $\operatorname{coin}\left(\tau_{\alpha} \varphi, \psi\right) \subset G$ for each $\alpha \in \Delta$ with $p(\operatorname{Coin}(\alpha \tilde{f}, \tilde{g}))$ an essential coincidence class.

Proof. By the equality $(*)$ in the proof of Lemma 2.2,

$$
\begin{aligned}
\bar{p}^{-1}(\operatorname{Coin}(f, g)) & =\coprod_{\bar{\alpha} \in \Delta / H} \operatorname{Coin}(\bar{\alpha} \bar{f}, \bar{g}) \\
& =\coprod_{\bar{\alpha} \in \Delta / H} \coprod_{[\gamma] \in \mathcal{R}\left[\tau_{\alpha} \varphi^{\prime}, \psi^{\prime}\right]} p^{\prime}(\operatorname{Coin}(\gamma \alpha \tilde{f}, \tilde{g})) .
\end{aligned}
$$

By Lemma 2.2.(2) and Corollary 4.3,

$$
\begin{aligned}
\operatorname{Coin}(f, g) & =\coprod_{[\bar{\alpha}] \in \mathcal{R}[\bar{\varphi}, \bar{\psi}]} \coprod_{[\gamma] \in \operatorname{im}\left(\hat{j}_{\alpha}\right)} p(\operatorname{Coin}(\gamma \alpha \tilde{f}, \tilde{g})) \\
\bar{p}^{-1} p(\operatorname{Coin}(\gamma \alpha \tilde{f}, \tilde{g})) & =\coprod_{\left[\Pi / G: u_{\gamma \alpha} \operatorname{coin}\left(\tau_{\gamma \alpha} \varphi, \psi\right)\right]} p^{\prime}\left(\operatorname{Coin}\left(\gamma^{\prime} \alpha \tilde{f}, \tilde{g}\right)\right) .
\end{aligned}
$$

Thus we have a relabelling of the set of all the coincidence classes of the liftings $(\bar{\alpha} \bar{f}, \bar{g})$ of $(f, g), \bar{\alpha} \in \Delta / H$ :

$$
\begin{aligned}
& \coprod_{\bar{\alpha} \in \Delta / H} \coprod_{[\gamma] \in \mathcal{R}\left[\tau_{\alpha} \varphi^{\prime}, \psi^{\prime}\right]} p^{\prime}(\operatorname{Coin}(\gamma \alpha \tilde{f}, \tilde{g})) \\
& \quad=\coprod_{[\bar{\alpha}] \in \mathcal{R}[\bar{\varphi}, \bar{\psi}]} \coprod_{[\gamma] \in \operatorname{im}\left(\hat{j}_{\alpha}\right)} \coprod_{\left[\Pi / G: u_{\gamma \alpha} \operatorname{coin}\left(\tau_{\gamma \alpha} \varphi, \psi\right)\right]} p^{\prime}\left(\operatorname{Coin}\left(\gamma^{\prime} \alpha \tilde{f}, \tilde{g}\right)\right) .
\end{aligned}
$$


Therefore,

$$
\begin{aligned}
\sum_{\bar{\alpha} \in \Delta / H} N(\bar{\alpha} \bar{f}, \bar{g}) & =\sum_{\bar{\alpha} \in \Delta / H} \sum_{[\gamma] \in \mathcal{R}\left[\tau_{\alpha} \varphi^{\prime}, \psi^{\prime}\right]} \epsilon_{\gamma \alpha}^{\prime} \sum_{\bar{\alpha} \in \mathcal{R}[\bar{\varphi}, \bar{\psi}]} \sum_{[\gamma] \in \operatorname{im}\left(\hat{j}_{\alpha}\right)} \sum_{\left[\Pi / G: u_{\gamma \alpha} \operatorname{coin}\left(\tau_{\gamma \alpha} \varphi, \psi\right)\right]} \epsilon_{\gamma^{\prime} \alpha}^{\prime} \\
& \leq \sum_{\bar{\alpha} \in \mathcal{R}[\bar{\varphi}, \bar{\psi}]} \sum_{[\gamma] \in \operatorname{im}\left(\hat{j}_{\alpha}\right)}\left[\Pi / G: u_{\gamma \alpha} \operatorname{coin}\left(\tau_{\gamma \alpha} \varphi, \psi\right)\right] \cdot \epsilon_{\gamma \alpha} \\
& \leq|\Pi / G| \sum_{\bar{\alpha} \in \mathcal{R}[\bar{\varphi}, \bar{\psi}]} \sum_{[\gamma] \in \operatorname{im}\left(\hat{j}_{\alpha}\right)} \epsilon_{\gamma \alpha} \\
& =|\Pi / G| N(f, g) .
\end{aligned}
$$

The inequalities above become equalities if and only if for each $\gamma \in H$ and $\bar{\alpha} \in \Delta / H$ with $p(\operatorname{Coin}(\gamma \alpha \tilde{f}, \tilde{g}))$ an essential coincidence class, we have $u_{\gamma \alpha} \operatorname{coin}\left(\tau_{\gamma \alpha} \varphi, \psi\right)=\{1\}$.

Corollary 4.7. If $\Pi=G$ in Theorem 4.6, then

$$
N(f, g)=\sum_{\bar{\alpha} \in \Delta / H} N(\bar{\alpha} \bar{f}, \bar{g}) \text {. }
$$

Proof. For any $\gamma \in H$ and $\alpha \in \Delta, \operatorname{coin}\left(\tau_{\gamma \alpha} \varphi, \psi\right) \subset \Pi=G$. Hence the equality follows from Theorem 4.6.

Let $L$ be a simply connected nilpotent Lie group and let $C$ a maximal compact subgroup of $\operatorname{Aut}(L)$. A discrete and cocompact subgroup $\Pi$ of $L \rtimes C \subset \operatorname{Aff}(L)=L \rtimes \operatorname{Aut}(L)$ is called an almost crystallographic group. Moreover, if $\Pi$ is torsion-free, then $\Pi$ is called an almost Bieberbach group and the quotient space $\Pi \backslash L$ an infra-nilmanifold. In particular, if $\Pi \subset L$, then $\Pi \backslash L$ is called a nilmanifold. Recall from [15] that $\Gamma=\Pi \cap L$ is the maximal normal nilpotent subgroup of $\Pi$ with finite quotient group $\Psi=$ $\Pi / \Gamma$, called the holonomy group of $\Pi \backslash L$. Thus the nilmanifold $\Gamma \backslash L$ is a finite regular covering of $\Pi \backslash L$. This nilmanifold $\Gamma \backslash L$ is called the nil-covering of $\Pi \backslash L$.

Now, suppose that $M$ and $N$ are infra-nilmanifolds of the same dimension and that $M_{G}$ and $N_{H}$ are nilmanifolds.

Lemma 4.8. Let $(f, g): M \rightarrow N$ be a pair of maps. For any $\alpha \in \Pi$ with $p(\operatorname{Coin}(\alpha \tilde{f}, \tilde{g}))$ an essential coincidence class, if there exists $\alpha^{\prime} \in[\alpha] \in$ $\mathcal{R}[\varphi, \psi]$ such that $p^{\prime}\left(\operatorname{Coin}\left(\alpha^{\prime} \tilde{f}, \tilde{g}\right)\right)$ is essential, then $\operatorname{coin}\left(\tau_{\alpha} \varphi, \psi\right)=\{1\}$. 
Proof. Since $p^{\prime}\left(\operatorname{Coin}\left(\alpha^{\prime} \tilde{f}, \tilde{g}\right)\right)$ is essential, it follows that $\operatorname{coin}\left(\tau_{\alpha} \varphi^{\prime}, \psi^{\prime}\right)=$ $\{1\}$, see [6]. Since $\Pi$ is torsion-free and $\Pi / G$ is finite, we obtain that $\operatorname{coin}\left(\tau_{\alpha} \varphi, \psi\right)=\{1\}$ from the exact sequence

$$
1 \longrightarrow \operatorname{coin}\left(\tau_{\alpha} \varphi^{\prime}, \psi^{\prime}\right) \stackrel{i_{\alpha}}{\longrightarrow} \operatorname{coin}\left(\tau_{\alpha} \varphi, \psi\right) \stackrel{u_{\alpha}}{\longrightarrow} \operatorname{coin}\left(\tau_{\bar{\alpha}} \bar{\varphi}, \bar{\psi}\right) .
$$

TheOREm 4.9. Let $(f, g): M \rightarrow N$ be a pair of maps between orientable infra-nilmanifolds of the same dimension. Suppose that $f_{*}(G) \subset H$ and $g_{*}(G) \subset H$. Then

$$
N(f, g)=\frac{1}{[\Pi: G]} \sum_{\bar{\alpha} \in \Delta / H} N(\bar{\alpha} \bar{f}, \bar{g}) .
$$

Proof. Suppose that $p(\operatorname{Coin}(\alpha \tilde{f}, \tilde{g})), \alpha \in \Pi$, is an essential coincidence class and that $p^{\prime}\left(\operatorname{Coin}\left(\alpha^{\prime} \tilde{f}, \tilde{g}\right)\right), \alpha^{\prime} \in[\alpha] \in \mathcal{R}[\varphi, \psi]$, is an inessential coincidence class. Then for each free element $x \in p(\operatorname{Coin}(\alpha \tilde{f}, \tilde{g}))$, all elements in $\bar{p}^{-1}(x) \cap p^{\prime}\left(\operatorname{Coin}\left(\alpha^{\prime} \tilde{f}, \tilde{g}\right)\right)$ are not free in $p^{\prime} \operatorname{Coin}\left(\alpha^{\prime} \tilde{f}, \tilde{g}\right)$. This implies that $x$ must be a self-reducing element and thus the coincidence class $p(\operatorname{Coin}(\alpha \tilde{f}, \tilde{g}))$ is defective. However, there are no defective classes in orientable manifolds. Thus our result follows from Lemma 4.8 and Theorem 4.6.

\section{$\S 5$. Two fold orientable covering}

Let $M$ and $N$ be closed non-orientable smooth manifolds of the same dimension, and $(f, g): M \rightarrow N$ a pair of transversal maps. Let $\Pi$ and $\Delta$ be the groups of covering transformations on the universal coverings $\widetilde{M}$ and $\widetilde{N}$ of $M$ and $N$, respectively. Let $\Pi^{+}$and $\Delta^{+}$be the subgroups of $\Pi$ and $\Delta$, respectively, which consist of all orientation preserving elements and let $\bar{p}: \bar{M} \rightarrow M$ and $\bar{q}: \bar{N} \rightarrow N$ be the two fold orientable coverings of $M$ and $N$, respectively. In this section, let us suppose that $f, g$ admit liftings $\bar{f}, \bar{g}: \bar{M} \rightarrow \bar{N}$, i.e.,

$$
\varphi\left(\Pi^{+}\right) \subset \Delta^{+}, \quad \psi\left(\Pi^{+}\right) \subset \Delta^{+} .
$$

Recall from Theorem 4.6 that

$$
N(f, g) \geq \frac{1}{2} \sum_{\bar{\alpha} \in \Delta / \Delta^{+}} N(\bar{\alpha} \bar{f}, \bar{g}),
$$


and equality occurs if and only if it holds $\operatorname{coin}\left(\tau_{\alpha} \varphi, \psi\right) \subset \Pi^{+}$for each $\alpha \in \Delta$ with $p(\operatorname{Coin}(\alpha \tilde{f}, \tilde{g}))$ an essential coincidence class.

On the other hand, recalling from Lemma 3.8, we see that

$$
\begin{aligned}
& p(\operatorname{Coin}(\alpha \tilde{f}, \tilde{g})) \text { is defective } \\
& \Longleftrightarrow \operatorname{coin}\left(\tau_{\alpha} \varphi, \psi\right) \cap \Pi^{+} \neq \operatorname{coin}\left(\tau_{\alpha} \varphi, \psi\right) \cap\left(\tau_{\alpha} \varphi\right)^{-1}\left(\Delta^{+}\right) \\
& \Longleftrightarrow \operatorname{coin}\left(\tau_{\alpha} \varphi, \psi\right) \nsubseteq \Pi^{+} \text {and } \tau_{\alpha} \varphi(\Pi) \subset \Delta^{+} .
\end{aligned}
$$

Thus we have the following result.

Proposition 5.1. The condition that all of the essential coincidence classes of $(f, g)$ are not defective is a necessary condition for the equality

$$
N(f, g)=\frac{1}{2} \sum_{\bar{\alpha} \in \Delta / \Delta^{+}} N(\bar{\alpha} \bar{f}, \bar{g}) .
$$

Now, we suppose further that $\varphi(\Pi) \subset \Delta^{+}$and $\psi(\Pi) \subset \Delta^{+}$. Then we have the commuting diagram

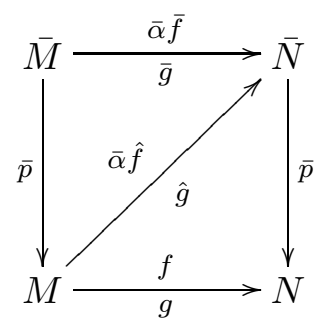

By Corollary 4.7, we have

$$
N(f, g)=\sum_{\bar{\alpha} \in \Delta / \Delta^{+}} N(\bar{\alpha} \hat{f}, \hat{g})
$$

In particular, $\mathbb{S}=p(\operatorname{Coin}(\alpha \tilde{f}, \tilde{g})) \subset \operatorname{Coin}(f, g)$ is essential if and only if $\hat{\mathbb{S}}_{\bar{\alpha}}=p(\operatorname{Coin}(\alpha \tilde{f}, \tilde{g})) \subset \operatorname{Coin}(\bar{\alpha} \hat{f}, \hat{g})$ is essential.

Recalling from Corollary 4.3 that

$$
\bar{p}^{-1} p(\operatorname{Coin}(\alpha \tilde{f}, \tilde{g}))=\coprod_{\left[\Pi / \Pi^{+}: u_{\alpha} \operatorname{coin}\left(\tau_{\alpha} \varphi, \psi\right)\right]} p^{\prime}\left(\operatorname{Coin}\left(\alpha^{\prime} \tilde{f}, \tilde{g}\right)\right)
$$


we see that

$$
\begin{aligned}
\mathbb{S}= & p(\operatorname{Coin}(\alpha \tilde{f}, \tilde{g})) \text { is defective } \\
\Longleftrightarrow & \operatorname{coin}\left(\tau_{\alpha} \varphi, \psi\right) \cap \Pi^{+} \neq \operatorname{coin}\left(\tau_{\alpha} \varphi, \psi\right) \cap\left(\tau_{\alpha} \varphi\right)^{-1}\left(\Delta^{+}\right) \\
\Longleftrightarrow & \operatorname{coin}\left(\tau_{\alpha} \varphi, \psi\right) \nsubseteq \Pi^{+} \\
\Longleftrightarrow & u_{\alpha} \operatorname{coin}\left(\tau_{\alpha} \varphi, \psi\right)=\Pi / \Pi^{+} \\
\Longleftrightarrow & \text { the coincidence class } \mathbb{S}=p(\operatorname{Coin}(\alpha \tilde{f}, \tilde{g})) \text { is covered by } \\
& \quad \text { a unique coincidence class } \overline{\mathbb{S}}=p^{\prime}\left(\operatorname{Coin}\left(\alpha^{\prime} \tilde{f}, \tilde{g}\right)\right),
\end{aligned}
$$

and $\mathbb{S}=p(\operatorname{Coin}(\alpha \tilde{f}, \tilde{g}))$ is not defective if and only if $\mathbb{S}$ is covered by two coincidence classes $\overline{\mathbb{S}}_{1}=p^{\prime}\left(\operatorname{Coin}\left(\alpha^{\prime} \tilde{f}, \tilde{g}\right)\right)$ and $\overline{\mathbb{S}}_{2}=p^{\prime}\left(\operatorname{Coin}\left(\alpha^{\prime \prime} \tilde{f}, \tilde{g}\right)\right)$ for some $\alpha^{\prime}, \alpha^{\prime \prime} \in[\alpha]$.

It is easy to observe the following, cf. [18, Corollaries 4.2 and 4.3].

COROLlaRY 5.2. Under the notation above, we have:

(1) If $\mathbb{S}$ is not defective, then

$$
\operatorname{ind}\left(\bar{\alpha} \bar{f}, \bar{g} ; \overline{\mathbb{S}}_{1}\right)=-\operatorname{ind}\left(\bar{\alpha} \bar{f}, \bar{g} ; \overline{\mathbb{S}}_{2}\right), \quad|\operatorname{ind}|(f, g ; \mathbb{S})=|\operatorname{ind}|\left(\bar{\alpha} \bar{f}, \bar{g} ; \overline{\mathbb{S}}_{i}\right)
$$

(2) If $\mathbb{S}$ is defective, then $\operatorname{ind}(\bar{\alpha} \bar{f}, \bar{g} ; \overline{\mathbb{S}})=0$.

COROLlary 5.3. Under the notation above, we have:

(1) $L(\bar{\alpha} \bar{f}, \bar{g})=0$.

(2) $N(\bar{\alpha} \bar{f}, \bar{g})$ is even.

(3) $N(f, g) \geq \frac{1}{2} \sum_{\bar{\alpha} \in \Delta / \Delta^{+}} N(\bar{\alpha} \bar{f}, \bar{g})$, and the equality holds if and only if all essential coincidence classes of $(f, g)$ are not defective.

(4) $N(\bar{\alpha} \bar{f}, \bar{g})=0$ if and only if all essential coincidence classes of $(\bar{\alpha} \hat{f}, \hat{g})$ are defective.

In the rest of this section, we consider the case of non-orientable infranilmanifolds with holonomy group $\mathbb{Z}_{2}$.

THEOREM 5.4. Let $M=\Pi \backslash L$ be a non-orientable infra-nilmanifold with holonomy group $\Psi=\mathbb{Z}_{2}$. Then for any pair of self maps $(f, g): M \rightarrow$ 
$M$ which admit liftings $\bar{f}, \bar{g}: \bar{M} \rightarrow \bar{M}$ on the nil-covering $\bar{M}$ of $M$,

$$
\begin{aligned}
N(f, g) & =\frac{1}{2}(N(\bar{f}, \bar{g})+N(\bar{\alpha} \bar{f}, \bar{g})) \\
& =\frac{1}{2}(|L(\bar{f}, \bar{g})|+|L(\bar{\alpha} \bar{f}, \bar{g})|) \\
& =\frac{1}{|\Psi|} \sum_{A \in \Psi}\left|\operatorname{det}\left(G_{*}-A_{*} F_{*}\right)\right|,
\end{aligned}
$$

where $A_{*}, F_{*}, G_{*}$ are the linear transformations on the Lie algebra $\mathfrak{L}$ of $L$ induced by $A, f, g$, respectively.

Proof. Suppose that $\varphi(\Pi) \nsubseteq \Pi^{+}$or $\psi(\Pi) \nsubseteq \Pi^{+}$, and suppose that there exists an essential coincidence class $p \operatorname{Coin}(\alpha \tilde{f}, \tilde{g})$ with $\operatorname{coin}\left(\tau_{\alpha} \varphi, \psi\right) \nsubseteq$ $\Pi^{+}$. Then $u_{\alpha} \operatorname{coin}\left(\tau_{\alpha} \varphi, \psi\right)=\Pi / \Pi^{+}$. We may assume that $f$ and $g$ are transversal so that $p(\operatorname{Coin}(\alpha \tilde{f}, \tilde{g}))$ consists of one point $x$. Since $\varphi(\Pi) \nsubseteq \Pi^{+}$ or $\psi(\Pi) \nsubseteq \Pi^{+}$and $\operatorname{coin}\left(\tau_{\alpha} \varphi, \psi\right) \nsubseteq \Pi^{+}$, we have $\left(\tau_{\bar{\alpha}} \bar{\varphi}\right)^{-1}(\overline{1})=\overline{1}$. Thus, Lemma 3.9 implies that there is $\beta \notin \Pi^{+}$such that the coincidence class $p^{\prime}(\operatorname{Coin}(\alpha \tilde{f}, \tilde{g}))=\{\bar{x}, \bar{\beta} \bar{x}\}$ and $\bar{x}$ and $\bar{\beta} \bar{x}$ do not reduce one another. Hence $p^{\prime}(\operatorname{Coin}(\alpha \tilde{f}, \tilde{g}))$ is essential. But this is a contradiction, $\operatorname{since} \operatorname{coin}\left(\tau_{\alpha} \varphi, \psi\right)=$ $\{1\} \subset \Pi^{+}$by Lemma 4.8. Therefore whenever $p(\operatorname{Coin}(\alpha \tilde{f}, \tilde{g}))$ is an essential coincidence class, we have $\operatorname{coin}\left(\tau_{\alpha} \varphi, \psi\right) \subset \Pi^{+}$.

Suppose next that $\varphi(\Pi) \subseteq \Pi^{+}$and $\psi(\Pi) \subseteq \Pi^{+}$. We will show that all coincidence classes are inessential. Since $\bar{M}$ is a nilmanifold, $\Pi^{+}$is a nilpotent group. Thus $\gamma_{n}\left(\Pi^{+}\right)=\left[\gamma_{n-1}\left(\Pi^{+}\right), \Pi^{+}\right]=1$ for some integer $n$. Since $\Pi^{+}=\Pi \cap L$ is the maximal normal nilpotent subgroup of $\Pi$, $\Pi$ is not a nilpotent group and thus $\gamma_{n}(\Pi) \neq 1$. Let $K=\operatorname{ker} \varphi \cap \operatorname{ker} \psi$. Then $\gamma_{n}(\Pi) \subseteq K$ and this implies that the Hirsch rank of $K$ is bigger than 1. Let $r=\Pi \rightarrow \Pi / K$ be the natural projection. Then $\varphi$ and $\psi$ factor through $\Pi / K$ :

$$
\Pi \stackrel{r}{\longrightarrow} \Pi / K \underset{\psi^{\prime}}{\stackrel{\varphi^{\prime}}{\longrightarrow}} \Pi^{+} \subset \Pi .
$$

Since $K \subseteq \operatorname{ker} \varphi$ and $\Pi, \Pi^{+}$are torsion-free virtually polycyclic group, the quotient group $\Pi / K$ is a torsion-free virtually polycyclic group. By $[1$, Theorem 1] or [2, Theorem 1.2], there is a closed infra-solvmanifold $N$ with $\pi_{1}(N) \cong \Pi / K$. Since $M$ and $N$ are aspherical, there are maps

$$
M \stackrel{\pi}{\longrightarrow} N \underset{g^{\prime}}{\stackrel{f^{\prime}}{\longrightarrow}} M
$$


such that $f^{\prime} \circ \pi, g^{\prime} \circ \pi$ are homotopic to $f, g$ respectively. We may assume that $f^{\prime}, g^{\prime}$ are transversal. Since $\operatorname{dim} N<\operatorname{dim} M$, [3, Theorem II.15.2] induces that $\left(f^{\prime} \times g^{\prime}\right) \cap \Delta_{M}=\varnothing$, i.e., $\operatorname{Coin}\left(f^{\prime}, g^{\prime}\right)=\varnothing$. This implies that Coin $\left(f^{\prime} \circ \pi, g^{\prime} \circ \pi\right)=\varnothing$. Hence $f, g$ are homotopic to coincidence free maps. In all, we have shown that whenever $p \operatorname{Coin}(\alpha \tilde{f}, \tilde{g})$ is an essential coincidence class, we have $\operatorname{coin}\left(\tau_{\alpha} \varphi, \psi\right) \subset \Pi^{+}$. Hence the first equality follows from Theorem 4.6. The second equality is well-known (cf. [4], [10]) and the third equality follows from Theorem 2.4 of [12].

Remark 5.5. For every self map $f$ on the Klein bottle, there is a map $f^{\prime}$ on the Klein bottle such that $f^{\prime}$ is homotopic to $f$ and $f^{\prime}$ admits a lifting on the torus. As an application of Theorem 5.4, using the averaging formula we can easily compute the Nielsen coincidence number of any pair of self maps on the Klein bottle. In [5], Dobreńko and Jezierski showed the same result on the Klein bottle using the fiber structure of the Klein bottle. In the following section we will consider other examples.

\section{§6. Examples}

In this section we illustrate, by some examples, how practical the averaging formula on infra-nilmanifolds is.

EXAMPLE 6.1. Let

$$
a=\left[\begin{array}{c}
\frac{1}{2} \\
0 \\
0
\end{array}\right], \quad A=\left[\begin{array}{ccc}
1 & 0 & 0 \\
0 & -1 & 0 \\
0 & 0 & -1
\end{array}\right]
$$

Then $A$ has period 2 , and $(a, A)^{2}=\left(a+A a, I_{3}\right)=\left(\left[\begin{array}{l}1 \\ 0 \\ 0\end{array}\right], I_{3}\right) \in \mathbb{R}^{3} \rtimes \operatorname{Aut}\left(\mathbb{R}^{3}\right)$. Let $\Gamma$ be the integral matrices of $\mathbb{R}^{3}$. Then it forms a lattice in $\mathbb{R}^{3}$. It is easy to check that the subgroup

$$
\Pi=\langle\Gamma,(a, A)\rangle \subset \mathbb{R}^{3} \rtimes \operatorname{Aut}\left(\mathbb{R}^{3}\right)
$$

generated by the lattice $\Gamma$ and the element $(a, A)$ is discrete and torsion free. Furthermore, $\Gamma$ is a normal subgroup of $\Pi$ of index 2 . Thus $\Pi$ is an almost Bieberbach group. 
Let $D, E \in \operatorname{Aut}\left(\mathbb{R}^{3}\right)$ be given by

$$
D=\left[\begin{array}{lll}
3 & 0 & 0 \\
0 & 1 & 1 \\
0 & 1 & 2
\end{array}\right], \quad E=\left[\begin{array}{ccc}
1 & 0 & 0 \\
0 & 2 & -1 \\
0 & 1 & 0
\end{array}\right]
$$

It is not difficult to check that $D A=A D, E A=A E$ and the conjugation by $(\{0,0,0\}, D),(a, E) \in \mathbb{R}^{3} \rtimes \operatorname{Aut}\left(\mathbb{R}^{3}\right)$ map $\Pi$ into $\Pi$ (and $\Gamma$ into $\Gamma$ ). Thus, the affine maps $(\{0,0,0\}, D),(a, E): \mathbb{R}^{3} \rightarrow \mathbb{R}^{3}$ induce $\phi_{D}, \phi_{(a, E)}: \Gamma \backslash \mathbb{R}^{3} \rightarrow \Gamma \backslash \mathbb{R}^{3}$ and $\Phi_{D}, \Phi_{(a, E)}: \Pi \backslash \mathbb{R}^{3} \rightarrow \Pi \backslash \mathbb{R}^{3}$ so that the following diagram commutes:

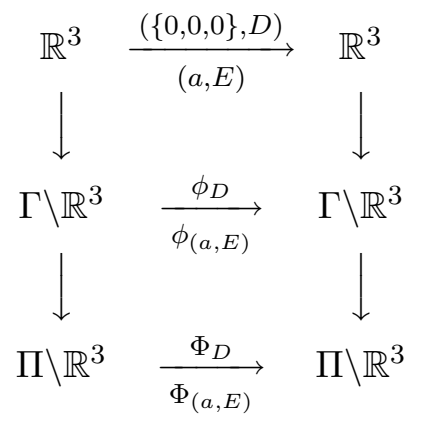

Note that all the vertical maps are the natural covering maps. In particular, $\Gamma \backslash \mathbb{R}^{3} \rightarrow \Pi \backslash \mathbb{R}^{3}$ is the nil-covering and the holonomy group of $\Pi \backslash \mathbb{R}^{3}$ is $\Psi=$ $\{I, A\} \cong \mathbb{Z}_{2}$. Thus by Theorem 5.4 , the Nielsen coincidence number of the maps $\Phi_{D}, \Phi_{(a, E)}: \Pi \backslash \mathbb{R}^{3} \rightarrow \Pi \backslash \mathbb{R}^{3}$ is:

$$
\begin{aligned}
N\left(\Phi_{D}, \Phi_{(a, E)}\right) & =\frac{1}{|\Psi|} \sum_{A \in \Psi}|\operatorname{det}(E-A D)| \\
& =\frac{1}{2}(|\operatorname{det}(E-D)|+|\operatorname{det}(E-A D)|) \\
& =\frac{1}{2}(|4|+|-12|)=8 .
\end{aligned}
$$

ExAMPLE 6.2. Let $L$ be the 3-dimensional Heisenberg group. That is,

$$
L=\left\{\left[\begin{array}{lll}
1 & x & z \\
0 & 1 & y \\
0 & 0 & 1
\end{array}\right]: x, y, z \in \mathbb{R}\right\} .
$$

We denote this general element by $\{x, y, z\}$. Let $\Gamma$ is the subgroup of $L$ which is generated by

$$
\{1,0,0\}, \quad\{0,1,0\}, \quad a=\left\{0,0, \frac{1}{2}\right\} .
$$


Then $\Gamma$ is a lattice of $L$.

Let $A: L \rightarrow L$ be the automorphism of $L$ given by

$$
A(\{x, y, z\})=\{-x,-y, z\} .
$$

Then $A$ has period 2, and $(a, A)^{2}=\left(a^{2}, I\right)=(\{0,0,1\}, I) \in L \rtimes \operatorname{Aut}(L)$, where $I$ is the identity automorphism of $L$. The subgroup

$$
\Pi=\langle\Gamma,(a, A)\rangle \subset L \rtimes \operatorname{Aut}(L)
$$

generated by the lattice $\Gamma$ and the element $(a, A)$ is discrete and torsion free, and $\Gamma$ is a normal subgroup of $\Pi$ of index 2 . Thus $\Pi$ is an almost Bieberbach group, $\Pi \backslash L$ is an infra-nilmanifold with holonomy group $\Psi=$ $\Pi / \Gamma=\{1, A\} \cong \mathbb{Z}_{2}$, and $\Gamma \backslash L \rightarrow \Pi \backslash L$ is the nil-covering.

Let $D, E: L \rightarrow L$ be the automorphisms of $L$ given by

$$
\begin{aligned}
& D(\{x, y, z\})=\left\{x+y, x,-z+\frac{1}{2} x^{2}+x y\right\}, \\
& E(\{x, y, z\})=\left\{y,-x+3 y, z-x y+\frac{3}{2} y^{2}\right\} .
\end{aligned}
$$

Clearly the conjugations by $(\{0,0,0\}, D),(\{0,0,0\}, E) \in L \rtimes \operatorname{Aut}(L)$ map $\Pi$ into $\Pi$ (and $\Gamma$ into $\Gamma)$. The affine maps $(\{0,0,0\}, D),(\{0,0,0\}, E)$ : $L \rightarrow L$ induce $\phi_{D}, \phi_{E}: \Gamma \backslash L \rightarrow \Gamma \backslash L$ and $\Phi_{D}, \Phi_{E}: \Pi \backslash L \rightarrow \Pi \backslash L$ so that the following diagram commutes:

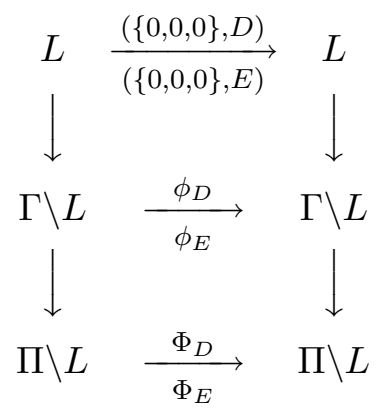

We take an ordered (linear) basis for the Lie algebra $\mathfrak{L}$ of $L$ as follows:

$$
\mathbf{e}_{1}=\left[\begin{array}{lll}
0 & 0 & 1 \\
0 & 0 & 0 \\
0 & 0 & 0
\end{array}\right], \quad \mathbf{e}_{2}=\left[\begin{array}{lll}
0 & 1 & 0 \\
0 & 0 & 0 \\
0 & 0 & 0
\end{array}\right], \quad \mathbf{e}_{3}=\left[\begin{array}{lll}
0 & 0 & 0 \\
0 & 0 & 1 \\
0 & 0 & 0
\end{array}\right] .
$$

With respect to this basis, the differentials of $A, D$ and $E$ are

$$
A_{*}=\left[\begin{array}{ccc}
1 & 0 & 0 \\
0 & -1 & 0 \\
0 & 0 & -1
\end{array}\right], \quad D_{*}=\left[\begin{array}{ccc}
-1 & 0 & 0 \\
0 & 1 & 1 \\
0 & 1 & 0
\end{array}\right], \quad E_{*}=\left[\begin{array}{ccc}
1 & 0 & 0 \\
0 & 0 & 1 \\
0 & -1 & 3
\end{array}\right] \text {. }
$$


Thus by Theorem 5.4, the Nielsen coincidence number of the maps $\Phi_{D}, \Phi_{E}$ : $\Pi \backslash L \rightarrow \Pi \backslash L$ is:

$$
\begin{aligned}
N\left(\Phi_{D}, \Phi_{E}\right) & =\frac{1}{|\Psi|} \sum_{A \in \Psi}\left|\operatorname{det}\left(E_{*}-A_{*} D_{*}\right)\right| \\
& =\frac{1}{2}\left(\left|\operatorname{det}\left(E_{*}-D_{*}\right)\right|+\left|\operatorname{det}\left(E_{*}-A_{*} D_{*}\right)\right|\right) \\
& =\frac{1}{2}(|-6|+|6|)=6 .
\end{aligned}
$$

Acknowledgement. The authors would like to thank the referee for making careful corrections of a few expressions in their original version.

\section{REFERENCES}

[1] L. Auslander and F. E. A. Johnson, On a conjecture of C. T. C. Wall, J. London Math. Soc. (2), 14 (1976), 331-332.

[2] O. Baues, Infra-solvmanifolds and rigidity of subgroups in solvable linear algebraic groups, Topology, 43 (2004), 903-924.

[3] G. E. Bredon, Topology and Geometry, Springer-Verlag, New York, 1993.

[4] R. Brooks and P. Wong, On changing fixed points and coincidences to roots, Proc. Amer. Math. Soc., 115 (1992), 527-533.

[5] R. Dobreńko and J. Jezierski, The coincidence Nielsen number on non-orientable manifolds, Rocky Mountain J. Math., 23 (1993), 67-85.

[6] D. L. Gonçalves, The coincidence Reidemeister classes of maps on nilmanifolds, Topol. Meth. Nonlin. Anal., 12 (1998), 375-386.

[7] D. L. Gonçalves and P. Wong, Nilmanifolds are Jiang-type spaces for coincidences, Forum Math., 13 (2001), 133-141.

[8] V. Guillemin and A. Pollack, Differential topology, Prentice-Hall, Englewood Cliffs, New Jersey, 1974.

[9] M. W. Hirsch, Differential topology, Graduate Text in Mathematics 33, SpringerVerlag, New York, 1976.

[10] J. Jezierski, The Nielsen number product formula for coincidences, Fund. Math., 134 (1990), 183-212.

[11] J. Jezierski, The semi-index product formula, Fund. Math., 140 (1992), 99-120.

[12] S. W. Kim and J. B. Lee, Anosov theorem for coincidences on nilmanifolds, Fund. Math., 185 (2005), no. 3, 247-259.

[13] S. W. Kim, J. B. Lee and K. B. Lee, Averaging formula for Nielsen numbers, Nagoya Math. J., 178 (2005), 37-53.

[14] J. B. Lee and K. B. Lee, Lefschetz numbers for continuous maps, and periods for expanding maps on infra-nilmanifolds, J. Geom. Phys., 56 (2006), 2011-2023.

[15] K. B. Lee and F. Raymond, Rigidity of almost crystallographic groups, Contemporary Math. Amer. Math. Soc., 44 (1985), 73-78. 
[16] C. K. McCord, Estimating Nielsen numbers on infrasolvmanifolds, Pacific J. Math., 154 (1992), 345-368.

[17] H. Schirmer, Mindestzahlen von Koinzidenpunkten, J. Reine Angew. Math., 194 (1955), 21-39.

[18] D. Vendrúscolo, Coincidence classes in nonorientable manifolds, Fixed Point Theory Appl. 2006, Special Issue, Art. ID 68513, 9 pp.

[19] P. Wong, Reidemeister number, Hirsch rank, coincidence on polycyclic groups and solvmanifolds, J. reine angew. Math., 524 (2000), 185-204.

Seung Won Kim

School of Mathematics

Korea Institute for Advanced Study

Seoul 130-722

Korea

swkim@kias.re.kr

Jong Bum Lee

Department of Mathematics

Sogang University

Seoul 121-742

Korea

jlee@sogang.ac.kr 